\title{
Acute type A dissection without intimal tear in arch: Proximal or extensive repair?
}

\author{
Hao Zhang, MD, Xilong Lang, MD, Fanglin Lu, MD, Zhigang Song, MD, Jun Wang, MD, \\ Lin Han, MD, and Zhiyun Xu, MD
}

\begin{abstract}
Objective: For acute type A dissection without an intimal tear in the arch, the optimal surgical strategy is unknown. The present study was designed to clarify the issue by comparing the early and late outcomes of proximal (PR) and extensive repair (ER).
\end{abstract}

\begin{abstract}
Methods: From January 2002 to June 2010, 331 patients with acute type A dissection were treated surgically at our institute. Of these 331 patients, 197 were identified without an arch tear on the preoperative imaging examination and by intraoperative inspection. Of these 197 patients, 74 underwent proximal repair, including the aortic root, ascending aortic, or hemiarch repair, and 88 underwent extensive repair, including proximal repair, total arch replacement and a stented elephant trunk technique. The perioperative variables and late results were statistically analyzed.
\end{abstract}

Results: No significant difference was found in the rates of early mortality and morbidity between the 2 groups, despite the shorter duration of circulatory arrest in the PR group. During long-term follow-up (mean, 55.7 \pm 33.1 months; maximum, 129), the overall survival rate in the whole cohort was $100 \%, 90.8 \%$, and $71.1 \%$ at 1,5 , and 8 years, respectively. No difference was found in survival between the 2 groups $(P>.05)$. However, complete thrombosis of the false lumen in the proximal descending aorta was achieved in $100 \%$ of the ER group and $24.6 \%$ of the PR group $(P<.001)$. For patients with a patent false lumen in the PR group, distal anastomosis leakage and unclosed small intimal tears were identified in $53.3 \%$ and $35.6 \%$ patients, respectively. The reintervention rate was also lower in the ER group than in the PR group $(4.9 \%$ vs $15.9 \%, P<.05)$ during follow-up. Moreover, the reintervention rate for patients with Marfan syndrome was $9.5 \%$ in the ER group and $38.5 \%$ in the PR group $(P<.05)$.

Conclusions: For patients with acute type A dissection without an intimal tear in the arch, extensive repair could promote the occlusion of distal false lumen and decrease the reintervention rate without increasing the operative risk. (J Thorac Cardiovasc Surg 2014;147:1251-5)

Of the cases of acute type A dissection (ATAD), only $10 \%-30 \%$ will include intimal tears in the $\operatorname{arch}^{1-3}$; thus, $70 \%-90 \%$ of patients with ATAD will have no tear in the arch. For this majority of patients with ATAD and an intact intimal, but dissected, aortic arch, the optimal surgical strategy for the aortic arch is still under debate. Proximal repair (PR) or extensive repair (ER) has been adopted by different centers; however, which one is optimal is unknown to date. PR consists of ascending aorta and hemiarch replacement, and ER is a more extensive strategy involving the aortic arch and descending aorta. It has traditionally been believed that PR will be sufficient

\footnotetext{
From the Institute of Cardiothoracic Surgery, Changhai Hospital, Second Military Medical University, Shanghai, China.

Disclosures: Authors have nothing to disclose with regard to commercial support.

Received for publication Jan 15, 2013; revisions received April 11, 2013; accepted for publication April 19, 2013; available ahead of print June 17, 2013.

Address for reprints: Zhiyun Xu, MD, Division of Cardiothoracic Surgery, Changhai Hospital, Second Military Medical University, 168 Changhai Rd, Shanghai, China (E-mail: zhiyunx@hotmail.com).

$0022-5223 / \$ 36.00$

Copyright (c) 2014 by The American Association for Thoracic Surgery

http://dx.doi.org/10.1016/j.jtcvs.2013.04.029
}

for saving the patient's life. ${ }^{4-6}$ However, it leaves the residual dissection in the distal aorta untreated, exposing patients to the risk of aortic rupture or reoperation. ${ }^{7-10} \mathrm{In}$ contrast, ER could obliterate the false lumen and decrease late aortic events. ${ }^{11-13}$ Nevertheless, ER has been considered to carry increased operative risk. ${ }^{4-6,14}$

The present retrospective study was designed to clarify this issue by comparing the early and late results of the 2 strategies for patients with ATAD without an arch tear (ATAD-wat).

\section{METHODS}

From January 2002 to June 2010, 331 patients with ATAD were treated surgically in our division. Of the 331 patients, 197 were identified without an arch tear using preoperative computed tomographic angiography, echocardiography, and intraoperative inspection under direct vision. Of the 197 patients, 74 underwent proximal aortic repair (PR), including aortic root, ascending aortic, or hemiarch repair, and 88 underwent extensive repair, including PR, total arch replacement, and a stented elephant trunk technique. The data were retrospectively collected from the database for the Division of Cardiothoracic Surgery, which was approved by the institutional review board of Changhai Hospital. The need for informed consent was waived for the present retrospective study. The surgical procedures were performed by 2 of us (Z.X. and L.H.). 


$$
\begin{aligned}
& \text { Abbreviations and Acronyms } \\
& \begin{aligned}
\text { ATAD } & =\text { acute type A dissection } \\
\text { ATAD-wat } & =\text { ATAD without an arch tear } \\
\text { DAL } & =\text { distal anastomosis leakage } \\
\text { ER } & =\text { extensive repair } \\
\text { FL } & =\text { false lumen } \\
\text { MFS } & =\text { Marfan syndrome } \\
\text { PR } & =\text { proximal repair }
\end{aligned}
\end{aligned}
$$

\section{Surgical Procedure}

A median sternotomy was used in all patients. Cannulation of femoral artery was used for cardiopulmonary bypass, the right axillary artery for antegrade cerebral perfusion, and the superior vena cava for retrograde cerebral perfusion. Cardioplegia was perfused through the coronary ostia after crossclamping. If necessary, aortic root procedures were performed subsequently.

Once an adequate core temperature was reached $\left(20^{\circ} \mathrm{C}-22^{\circ} \mathrm{C}\right.$ before 2004 and $26^{\circ} \mathrm{C}-28^{\circ} \mathrm{C}$ from 2004 to the end of the study period), circulatory arrest was initiated, with cerebral perfusion administered by retrograde cerebral perfusion before 2003 or antegrade cerebral perfusion after 2003. The arch, the origins of the 3 branch arteries, and the proximal descending aorta were inspected for an intimal tear under direct vision after removing the aortic clamp.

For patients undergoing PR, a prosthetic graft was used for ascending aorta or hemiarch replacement. For patients undergoing ER, the trunk of a 4-branch prosthetic graft (Boston Scientific Inc, Boston, Mass) was anastomosed to the proximal end of the native aorta. Next, a stent graft (MicroPort Medical, Co, Ltd, Shanghai, China) was inserted into the true lumen of the distal aorta through the arch incision. To determine the appropriate size of the stent graft, a ball-shaped valve sizer was inserted into the true lumen of the proximal descending aorta from the transverse incision to measure the exact inner diameter of the true lumen. The distal aorta incorporating the stent graft was securely anchored to the distal trunk of the 4-branch prosthetic graft, with antegrade blood perfusion of the lower body by way of 1 of the branches. During rewarming, the brachiocephalic arteries were anastomosed in an orderly fashion to the branches of the prosthetic graft.

\section{Follow-up}

The follow-up data were obtained by clinical interviews and a postal questionnaire. To evaluate the residual false lumen of the downstream aorta, contrast computed tomography of the aorta was obtained before discharge and during the follow-up period.

\section{Statistical Analysis}

Categorical variables are presented as frequencies and were analyzed using the chi-square test. Continuous variables are expressed as the mean \pm standard deviation, and normally distributed variables were analyzed using the Student $t$ test. Non-normal continuous variables were analyzed using the Kruskal-Wallis nonparametric test. Long-term survival was analyzed using the Kaplan-Meier method. Comparisons between groups were made using the log-rank test. $P<.05$ was considered significant.

\section{RESULTS}

\section{Preoperative Demographics}

The preoperative demographics of the 2 groups were similar and are listed in Table 1.

\section{Intra- and Postoperative Details}

The intra- and postoperative variables of the 2 groups are listed in Tables 2 and 3. The duration of crossclamping and circulatory arrest was shorter in the PR group than in the ER group $(P<.05)$. No differences were seen in the cardiopulmonary bypass duration or the incidence of blood transfusion. No differences were found in the number of concomitant procedures between the 2 groups. No intraoperative deaths occurred in the whole cohort. A total of $4(5.4 \%)$ and $5(5.7 \%)$ inhospital deaths occurred in the PR and ER groups, respectively $(P=.94)$. No significant differences were found in the rates of perioperative re-exploration for bleeding, prolonged ventilation, heart arrest, stroke, or renal failure between the 2 groups. No spinal cord injury was observed in any patient.

\section{Long-Term Results}

Survival. Of the cohort, 154 patients were discharged, and the follow-up data were complete for $139(90.3 \%)$. The mean follow-up period was $55.7 \pm 33.1$ months (maximum, 129). During the follow-up period, 3 and 0 aortic-related deaths occurred in the PR and ER groups, respectively $(P=.06)$. Other deaths were attributed to noncardiac diseases. The survival function (excluding in-hospital deaths) of the whole cohort is shown in Figure 1. The overall survival was $100 \%, 90.8 \%$, and $84.5 \%$ at 1,5 , and 8 years, respectively. The corresponding survival rates were $100 \%$, $85.6 \%$, and $80.5 \%$ in the PR group and $100 \%, 95.0 \%$, and $87.7 \%$ in the ER group. No difference was found in survival function between the 2 groups $(P=.11$; Figure 2$)$.

Behavior of false lumen. Computed tomographic images were obtained before discharge and 6-12 months after surgery in 69 patients in the PR group and 82 in the ER group. The computed tomographic findings at 6-12 months postoperatively revealed complete thrombosis in the false lumen (FL) at the level of proximal descending aorta in all patients in the ER group. However, complete thrombosis was observed in only $24(34.8 \%)$ and $17(24.6 \%)$ of the FLs at the level of the arch and proximal descending aorta in the patients in the PR group, respectively. At the diaphragmatic level, 14 $(20.3 \%)$ in the PR and $41(50 \%)$ in the ER group showed complete thrombosis, respectively $(P<.001$; Table 4$)$.

For 45 patients without thrombosis formation in the FL at the arch in the PR group 6-12 months after surgery, distal anastomosis leakage (DAL) was identified by computed tomography of the aorta in 24 patients $(53.3 \%)$ and an intimal tear of a residual FL was found in 7 and 3 patients with an intimal tear at the origin of the innominate artery and left subclavian artery, respectively. Six patients presented with an intimal tear at the proximal descending aorta (Table 5). In addition, multiple intimal tears were detected in 5 patients.

\section{Reintervention}

During follow-up, 11 of 65 patients $(16.9 \%)$ in the PR group underwent total arch replacement and the stented 
TABLE 1. Preoperative details

\begin{tabular}{lccc}
\hline \multicolumn{1}{c}{ Variable } & $\begin{array}{c}\text { PR group } \\
(\mathbf{n}=\mathbf{7 4})\end{array}$ & $\begin{array}{c}\text { ER group } \\
(\mathbf{n}=\mathbf{8 8})\end{array}$ & $\boldsymbol{P}$ value \\
\hline Age (y) & $49.1 \pm 12.6$ & $45.5 \pm 13.5$ & .45 \\
Male gender & $55(74.3)$ & $74(84.1)$ & .12 \\
MFS & $13(17.6)$ & $21(23.9)$ & .22 \\
LV ejection fraction (\%) & $59.2 \pm 7.1$ & $57.5 \pm 6.4$ & .11 \\
Diabetes mellitus & $4(5.3)$ & $4(4.5)$ & .80 \\
Hypertension & $47(63.5)$ & $64(72.7)$ & .14 \\
Renal dysfunction & $6(8.1)$ & $3(3.4)$ & .19 \\
Cardiac tamponade & $25(28.4)$ & $17(23.0)$ & .43 \\
Pleural effusion & $29(39.2)$ & $34(38.6)$ & .94 \\
Stroke & $2(2.7)$ & $1(1.1)$ & .46 \\
Hypoxemia & $17(23.0)$ & $16(18.2)$ & .45 \\
Aortic regurgitation & & & \\
$\quad$ Mild & $19(25.7)$ & $21(23.9)$ & .79 \\
Moderate & $14(18.9)$ & $20(22.7)$ & .55 \\
Severe & $21(28.4)$ & $19(21.6)$ & .32 \\
\hline
\end{tabular}

Data presented as mean \pm standard deviation or $\mathrm{n}(\%) . P R$, Proximal repair; $E R$, extensive repair; $M F S$, Marfan syndrome; $L V$, left ventricular.

elephant trunk technique for significant dilation of a patent FL in the arch and descending aorta. Five of them presented with Marfan syndrome (MFS). One patient with MFS died of severe sepsis 67 days after the reoperation, although the others survived. In contrast, 4 of 74 patients $(5.4 \% ; 2$ with MFS) underwent repeat intervention with thoracic endovascular aortic repair in the ER group for dilation of the FL in the descending aorta distal to the stent graft. All 4 patients were discharged without incidence. The difference in the reintervention rate was significant between the 2 groups $(P=.03)$. Furthermore, 5 of the 13 patients $(38.5 \%)$ with MFS in the PR group and 2 of the 21 $(9.5 \%)$ with MFS in the ER group required reintervention $(P=.04)$.

TABLE 2. Operative details and concomitant procedures

\begin{tabular}{lccr}
\hline \multicolumn{1}{c}{ Variable } & $\begin{array}{c}\text { PR group } \\
(\mathbf{n}=\mathbf{7 4})\end{array}$ & $\begin{array}{c}\text { ER group } \\
(\mathbf{n}=\mathbf{8 8})\end{array}$ & $\boldsymbol{P}$ value \\
\hline CPB time (min) & $179.7 \pm 39.5$ & $182.4 \pm 34.3$ & .64 \\
Cross-clamp time (min) & $102.4 \pm 30.3$ & $113.4 \pm 25.8$ & .01 \\
Duriation of CA (min) & $28.1 \pm 10.1$ & $35.4 \pm 11.6$ & $<.01$ \\
Retrograde perfusion & $8(10.8)$ & $7(8.0)$ & .53 \\
Antegrade perfusion & $66(89.2)$ & $81(92.0)$ & .53 \\
Total pRBCs (unit) & $15.6 \pm 7.1$ & $17.3 \pm 8.0$ & .16 \\
Concomitant procedures & & & \\
Bentall & $25(33.8)$ & $29(33.0)$ & .91 \\
Cabrol & $3(4.1)$ & $4(4.5)$ & .88 \\
AV replacement & $4(5.4)$ & $3(3.4)$ & .53 \\
David or partial David & $5(6.8)$ & $4(4.5)$ & .54 \\
MV repair/replacement & $7(9.5)$ & $9(10.2)$ & .87 \\
CABG & $8(10.8)$ & $8(9.1)$ & .72 \\
\hline
\end{tabular}

Data presented as mean \pm standard deviation or n (\%). $P R$, Proximal repair; $E R$, extensive repair; $C P B$, cardiopulmonary bypass; $C A$, circulation arrest; $p R B C s$, packed red blood cells; $A V$, aortic valve; $M V$, mitral valve; $C A B G$, coronary artery bypass grafting.
TABLE 3. Mortality and morbidity

\begin{tabular}{lccc}
\hline \multicolumn{1}{c}{ Variable } & $\begin{array}{c}\text { PR group } \\
(\mathbf{n}=\mathbf{7 4})\end{array}$ & $\begin{array}{c}\text { ER group } \\
(\mathbf{n = 8 8 )}\end{array}$ & $\boldsymbol{P}$ value \\
\hline Hospital mortality & $4(5.4)$ & $5(5.7)$ & .94 \\
Reexploration for bleeding & $2(2.7)$ & $4(4.5)$ & .54 \\
Ventilation $>$ 72 h & $15(20.3)$ & $17(19.3)$ & .88 \\
Heart arrest & $1(1.4)$ & $1(1.1)$ & .90 \\
New-onset stroke & $1(1.4)$ & $2(2.3)$ & .67 \\
Renal failure & $1(1.4)$ & $2(2.3)$ & .67 \\
Transient neurologic dysfunction & $8(10.8)$ & $11(12.5)$ & .74 \\
Infection or sepsis & $4(5.4)$ & $5(5.7)$ & .94 \\
\hline
\end{tabular}

Data presented as n (\%). $P R$, Proximal repair; $E R$, extensive repair.

\section{DISCUSSION}

ATAD remains a life-threatening disease, and emergency surgery is usually indicated. ${ }^{15}$ For patients with ATAD, the intimal tears can locate in the ascending aorta, aortic arch, and/or distal aorta. ${ }^{1-3}$ For patients with ATAD and an intimal tear in the arch, ER was required to obliterate the entry (or re-entry) and avoid dilation of the distal FL, according to the principle of resecting intimal tears.

Nevertheless, the optimal procedure for patients with ATAD-wat remains unknown. A very recent study of patients with ATAD-wat showed that ER could be applied without a greater perioperative risk than that with PR according to an analysis of the 30-day results. ${ }^{16}$ However, the long-term outcomes remain unclear. Therefore, we designed the present retrospective study to determine the optimal strategy for patients with ATAD-wat by comparing the early and late results between 2 strategies (ie, PR and ER).

The PR procedure performed in the present study consisted of resecting the primary intimal tear in the ascending

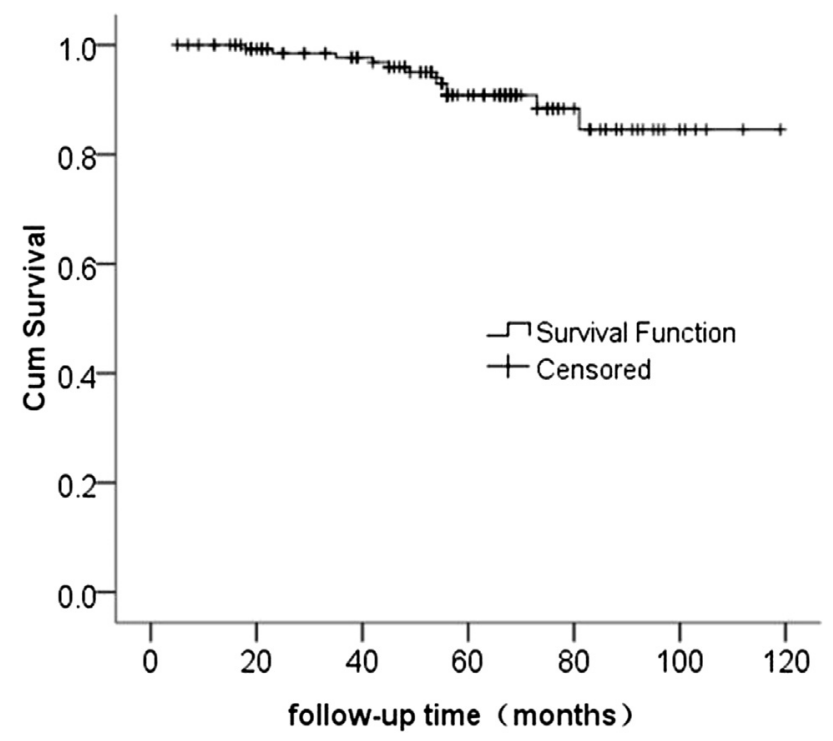

FIGURE 1. Overall survival function of patients with ATAD-wat from the whole cohort. 


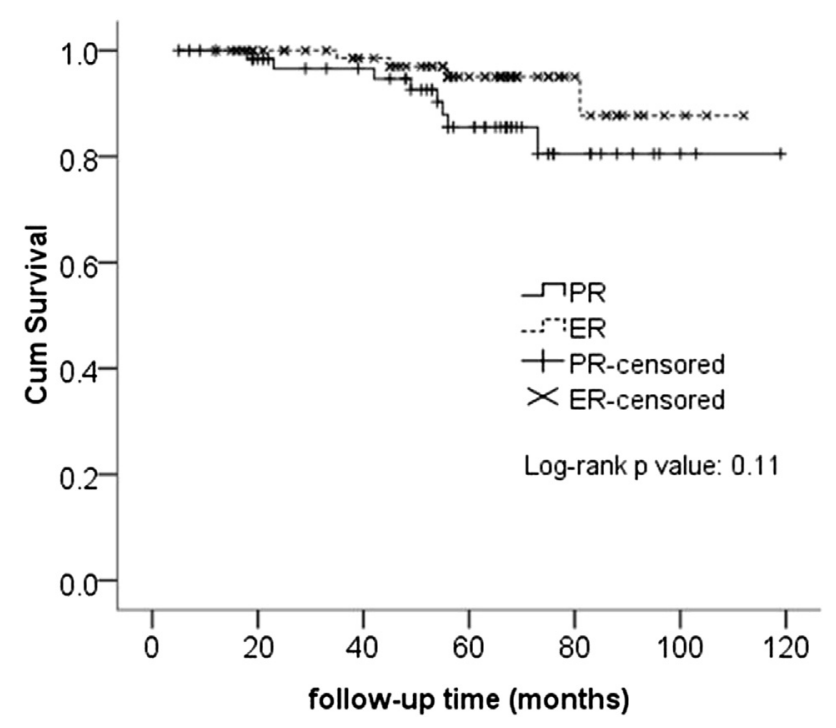

FIGURE 2. Survival of patients with ATAD-wat in the proximal repair $(P R)$ and extensive repair $(E R)$ groups during follow-up. No significant difference was found in survival between the 2 groups $(P=.11)$.

aorta and anastomosing the residual arch to the distal end of the graft. In theory, the FL of the distal aorta would thrombose when the blood perfusion was interrupted in patients with ATAD-wat after PR. However, a patent FL at the arch and proximal descending aorta was found in $65.2 \%$ and $75.4 \%$ of the patients in the PR group 12 months after surgery. We reviewed the postoperative computed tomographic images of the patients with a patent FL of the arch to disclose the causes of the high rate of patent FLs in the PR group. DAL was observed in $53.3 \%$ of the patients, and small intimal tears were identified at the brachiocephalic arteries or proximal descending aorta in $35.6 \%$ of the patients. Thus, we have inferred that the absence of thrombosis in the FL could be attributed to the continuous perfusion of blood flow into the FL through DAL or unclosed intimal tears. These findings provide the explanation for the dilation of the FL in the long term for patients with ATAD-wat who underwent PR.

For patients undergoing ER, in contrast, the dissected arch was replaced, and DAL was avoided because the distal aorta incorporating the stent graft was firmly anchored to the end of the proximal graft. In addition, the tear in the

TABLE 4. Formation of complete thrombosis in FL during follow-up

\begin{tabular}{lccccc}
\hline & \multicolumn{2}{c}{ PR group $(\mathbf{n}=6 \mathbf{6 9})$} & & ER group $(\mathbf{n}=\mathbf{8 2})$ \\
\cline { 2 - 3 } \cline { 5 - 6 } \multicolumn{1}{c}{ Variable } & $\begin{array}{c}\text { Before } \\
\text { discharge }\end{array}$ & At 6-12 mo & $\begin{array}{c}\text { Before } \\
\text { discharge }\end{array}$ & At 6-12 mo \\
\hline Transverse arch & $12(17.4)$ & $24(34.8)$ & - & - \\
$\begin{array}{l}\text { Proximal descending } \\
\quad \text { aorta }\end{array}$ & $12(17.4)$ & $17(24.6)$ & $66(80.5)$ & $82(100)^{*}$ \\
Diaphragmatic level & $10(14.5)$ & $14(20.3)$ & $26(31.7)$ & $37(45.1)^{*}$ \\
\hline
\end{tabular}

Data presented as n $(\%) . F L$, False lumen; $P R$, proximal repair; $E R$, extensive repair. $* P<.001$, compared with data from PR group at 6-12 months.
TABLE 5. Causes of patent FL at aortic arch for patients in PR group

\begin{tabular}{lc}
\hline \multicolumn{1}{c}{ Cause } & Patients (n) \\
\hline DAL & $24(53.3)$ \\
Tear at origin of innominate artery & $7(15.6)$ \\
Tear at origin of left subclavian artery & $3(6.7)$ \\
Tear at proximal descending aorta & $6(13.3)$ \\
Multiple tears & $5(11.1)$ \\
\hline
\end{tabular}

Data in parentheses are percentages. $F L$, False lumen; $P R$, proximal repair; $D A L$, distal anastomosis leakage.

proximal descending aorta could be closed by stent grafting. With these technical advantages, complete thrombosis was observed in the FL of the proximal descending aorta in all patients in the ER group. This result is coherent with previous reports of the ER procedure for patients with ATAD. ${ }^{11,12,17-19}$ In accordance with the fate of FL, a significantly lower rate of reoperation was observed in the ER group than in the PR group $(5.4 \%$ vs $16.9 \%$, $P<.05)$ during our 10 -year follow-up period. This has clearly proved that the ER procedure can reduce the risk of reoperation for ATAD-wat in the long term.

Nevertheless, we also noted that only $33.2 \%$ of the patients undergoing ER and $11.5 \%$ undergoing PR achieved complete thrombosis in the FL at the diaphragmatic level. This indicates that frequent and continuous follow-up will be essential, regardless of the surgical strategy used.

MFS and young age have been demonstrated to be independent risk factors for reoperation for ATAD. ${ }^{8,20}$ In the present study, the reintervention rate for patients with MFS was $9.5 \%$ in the ER group. However, $38.5 \%$ of the patients with MFS required reintervention in the PR group $(P<.05)$. According to the postoperative computed tomographic findings of the aorta, DAL was identified in all patients with MFS who required reintervention after PR. The high prevalence of DAL could be attributed to the weak intimal and medial wall of the aorta in patients with MFS that was susceptible to being torn by the anastomotic continuous suture. This result definitely justified the necessity of ER for ATAD combined with MFS.

Uchida et $\mathrm{al}^{11}$ reported that the survival rate after 5 years was significantly higher for patients undergoing ER than for those undergoing PR $(95.3 \%$ vs $69.0 \%, P=.03)$. However, the difference in survival between the 2 groups in our cohort did not reach significance $(P=.11)$, although the survival rate for the ER group at 5 and 8 years was more favorable than that for the PR group $(95.0 \%$ vs $85.6 \%$ at 5 years and $87.7 \%$ vs $80.5 \%$ at 8 years). It could be expected that the difference would be significant with an increased number of patients and prolonged follow-up period.

Regarding the operative risk, the overall in-hospital mortality was $4.9 \%$, and no significant differences were found between the PR and ER groups $(5.4 \%$ vs $5.7 \%, P>.05)$. Additionally, no statistically significant difference was found in the rate of postoperative morbidity, including 
reexploration for bleeding, prolonged ventilation, cerebral complications, or renal failure between the 2 groups. No spinal cord injury occurred in the whole cohort. These favorable early results indicated that ER does not increase the surgical risk compared with PR for patients with ATAD-wat. The reduction in operative risk for ER can be attributed to the combined use of several advanced techniques in cerebral protection, arch reconstruction, and stent grafting in this cohort.

The limitation of the present comparative study was the lack of a prospective and randomized design. However, a rigorous, prospective randomization of surgical procedures would be impractical for several reasons, including the infeasibility of obtaining informed consent in an emergency situation and the heterogeneity of the patient population and pathologic features, which have been elaborated by Bonser et al. ${ }^{21}$

\section{CONCLUSIONS}

The present comparative study has clearly demonstrated that, for patients with ATAD-wat, PR leaves the patients at a high risk of reintervention owing to the occurrence of DAL and undetected or unclosed small tears in the arch. In contrast, ER could reduce the risk of reintervention by obliterating the entries and distal FL without increased surgical risk. Furthermore, ER should be used more actively for patients of MFS because of the vulnerable aortic wall.

\section{References}

1. Rehders TC, Ince H, Nienaber CA. Aortic dissection: from aetiology to therapeutic management. Medicine. 2006;34:296-301.

2. Takami Y, Tajima K, Kato W, Fujii K, Hibino M, Munakata H, et al. Can we predict the site of entry tear by computed tomography in patients with acute type A aortic dissection? Clin Cardiol. 2012;35:500-4.

3. Okita Y, Takamoto S, Ando M, Morota T, Yamaki F, Kawashima Y, et al. Surgery for aortic dissection with intimal tear in the transverse aortic arch. Eur J Cardiothorac Surg. 1996;10:784-90.

4. Westaby S, Saito S, Katsumata T. Acute type A dissection: conservative methods provide consistently low mortality. Ann Thorac Surg. 2002;73:707-13.

5. Shiono M, Hata M, Sezai A, Niino T, Yagi S, Negishi N. Validity of a limited ascending and hemiarch replacement for acute type A aortic dissection. Ann Thorac Surg. 2006;82:1665-9.
6. Ohtsubo S, Itoh T, Takarabe K, Rikitake K, Furukawa K, Suda H, et al. Surgical results of hemiarch replacement for acute type A dissection. Ann Thorac Surg. 2002;74:S1853-6.

7. Ergin MA, Phillips RA, Galla JD, Lansman SL, Mendelson DS, Quintana CS, et al. Significance of distal false lumen after type A dissection repair. Ann Thorac Surg. 1994;57:820-4.

8. Kirsch M, Soustelle C, Houël R, Hillion ML, Loisance D. Risk factor analysis for proximal and distal reoperations after surgery for acute type A aortic dissection. J Thorac Cardiovasc Surg. 2002;123:318-25.

9. Zierer A, Voeller RK, Hill KE, Kouchoukos NT, Damiano RJ Jr, Moon MR. Aortic enlargement and late reoperation after repair of acute type A aortic dissection. Ann Thorac Surg. 2007;84:479-86.

10. Kirsch M, Legras A, Bruzzi M, Louis N. Fate of the distal aorta after surgical repair of acute DeBakey type I aortic dissection: a review. Arch Cardiovasc Dis. 2011;104:125-30.

11. Uchida N, Shibamura H, Katayama A, Shimada N, Sutoh M, Ishihara H. Operative strategy for acute type a aortic dissection: ascending aortic or hemiarch versus total arch replacement with frozen elephant trunk. Ann Thorac Surg. 2009;87: 773-7.

12. Sun L, Qi R, Zhu J, Liu Y, Zheng J. Total arch replacement combined with stented elephant trunk implantation: a new "standard" therapy for type A dissection involving repair of the aortic arch? Circulation. 2011;123:971-8.

13. Kazui T, Washiyama N, Muhammad BA, Terada H, Yamashita K, Takinami M, et al. Extended total arch replacement for acute type A aortic dissection: experience with seventy patients. J Thorac Cardiovasc Surg. 2000;119:558-65.

14. Crawford ES, Kirklin JW, Naftel DC, Svensson LG, Coselli JS, Safi HJ. Surgery for acute dissection of ascending aorta: should the arch be included? J Thorac Cardiovasc Surg. 1992;104:46-59.

15. Setacci F, Sirignano P, de Donato G, Chisci E, Perulli A, Setacci C. Acute aortic dissection: natural history and classification. J Cardiovasc Surg (Torino). 2010; 51:641-6.

16. Easo J, Weigang E, Hölzl PP, Horst M, Hoffmann I, Blettner M, et al., GERAADA Study Group. Influence of operative strategy for the aortic arch in DeBakey type I aortic dissection: analysis of the German Registry for Acute Aortic Dissection Type A. J Thorac Cardiovasc Surg. 2012;144:617-23.

17. Pochettino A, Brinkman WT, Moeller P, Szeto WY, Moser W, Cornelius K, et al. Antegrade thoracic stent grafting during repair of acute DeBakey I dissection prevents development of thoracoabdominal aortic aneurysms. Ann Thorac Surg. 2009;88:482-9.

18. Jakob H, Tsagakis K, Tossios P, Massoudy P, Thielmann M, Buck T, et al. Combining classic surgery with descending stent grafting for acute DeBakey type I dissection. Ann Thorac Surg. 2008;86:95-101.

19. Tsagakis K, Pacini D, Di Bartolomeo R, Benedik J, Cerny S, Gorlitzer M, et al Arch replacement and downstream stent grafting in complex aortic dissection: first results of an international registry. Eur J Cardiothorac Surg. 2011;39:87-94

20. Geirsson A, Bavaria JE, Swarr D, Keane MG, Woo YJ, Szeto WY, et al. Fate of the residual distal and proximal aorta after acute type A dissection repair using a contemporary surgical reconstruction algorithm. Ann Thorac Surg. 2007;84: 1955-64.

21. Bonser RS, Ranasinghe AM, Loubani M, Evans JD, Thalji NM, Bachet JE, et al Evidence, lack of evidence, controversy, and debate in the provision and performance of the surgery of acute type A aortic dissection. J Am Coll Cardiol. 2011; 58:2455-74 